\title{
Proprioceptive awareness, information about response-reinforcement contingencies, and operant heart-rate control
}

\author{
THOMAS R. McCANNE \\ Northern Illinois University, DeKalb, Illinois 60115 \\ and \\ CURT A. SANDMAN \\ Ohio State University, Columbus, Ohio 43210
}

\begin{abstract}
Twenty subjects were conditioned to raise their operant heart rates during the presentation of one stimulus and to lower operant heart rate during the presentation of a second stimulus. A 2 by 2 factorial design was utilized to examine the effects of proprioceptive awareness [high overall score on the Automonic Perception Questionnaire (APQ) vs. low APQ score] and instructions about the response-reinforcement contingencies (informed subjects vs. uninformed subjects) on operant heartrate control. The results indicated that informed subjects achieved better control over operant heart rate than uninformed subjects. No effects attributable to APQ score were detected. It is suggested that proprioceptive awareness may influence heart-rate control when no external feedback is available (e.g., Bergman \& Johnson, 1971), or when external feedback is relatively weak (e.g., Blanchard, Young, \& McLeod, 1972), but information about contingencies of reinforcement appears to have more impact on heart-rate control than proprioceptive awareness when relatively powerful external feedback is available. The heart-rate results appeared to be relatively independent of changes in skin responding and cephalic pulse amplitude.
\end{abstract}

Recently, investigators interested in human operant heart-rate conditioning have begun examining the role of an individual's proprioceptive awareness of automatic functioning in acquiring control over heart-rate changes (e.g., Bergman \& Johnson, 1971; Blanchard, Young, \& McLeod, 1972; McFarland, 1975; Sirota, Schwartz, \& Shapiro, 1974). Other operant heart-rate conditioning studies have been reported in which an individual's awareness of the response-reinforcement contingencies has been examined by manipulating instructional set (e.g., Bergman \& Johnson, 1972; McCanne \& Sandman, 1975a). The latter studies have indicated that subjects informed of the specific reinforcement contingencies are able to produce larger operant heart-rate accelerations than subjects who are not informed of the contingencies. Results for operant heart-rate deceleration are not as clear, but suggest that informed subjects acquire control over decelerations somewhat faster than uninformed subjects (McCanne \& Sandman, 1975a).

However, the research on proprioceptive awareness of automatic responding has produced equivocal results. Bergman and Johnson (1971), utilizing scores on the Autonomic Perception Questionnaire (APQ)

Address reprint requests to: Thomas R. McCanne, Department of Psychology, Northern Illinois University, DeKalb, Illinois 60115. developed by Mandler, Mandler, and Uviller (1958), found that subjects with middle APQ scores achieved more control over heart-rate acceleration and deceleration than subjects with extreme high and low scores when no external heart-rate feedback was available. However, Blanchard, Young, and McLeod (1972), using scores derived from five APQ items that deal with awareness of heart activity, found that subjects low in awareness of heart activity were able to significantly raise and lower their operant heart rates when provided with feedback, while subjects high in awareness could neither accelerate nor decelerate. While these results are not directly contradictory, they suggest that relationships between heart-rate control and autonomic perception (as measured by the APQ) are not as clear-cut as might be expected.

More recently, Sirota et al. (1974), using two items from the APQ that deal with heart activity, found no relationship between awareness of heart activity and operant heart-rate control. Similarly, McFarland (1975), employing correlational techniques, noted no relationship between awareness of heart activity (measured by five APQ items) and operant heart-rate control. These two studies further indicate the apparent lack of impact of autonomic awareness on heart-rate control.

However, in all of the studies cited above, subjects 
were given specific instructions to try to control heari-rate changes. When subjects are given such explicit instructions, awareness of autonomic activity may contribute very little to the acquisition of heartrate control. If subjects are not informed that changes in heart rate are the desired responses, then awareness of autonomic activity may play an important role in determining heart-rate control.

The present study was undertaken to evaluate the relative contributions of both proprioceptive awareness of autonomic functioning and the instructions given to subjects about the response-reinforcement contingencies to control over operant heart-rate acceleration and deceleration. Changes in endosomatic skin potential (GSP), galvanic skin response (GSR), and cephalic pulse amplitude (CPA) were also recorded to determine the relationship of these modalities to operant heart rate.

\section{METHOD}

\section{General Design}

The statistical model for the study was a 2 by 2 by 2 by 2 by 20 factorial design with repeated measures over the last three factors. The between-subject factors were APQ Score (see below for methods used to split subjects into two groups) and Instructional Set (informed subjects vs. uninformed subjects). There were five subjects in each of the four cells. The within-subject factors were: Training (acceleration training vs. deceleration training), Response Sample (pretrial response vs. trial response), and Trials (20 trials).

\section{Subjects}

Subjects were 7 male and 13 female students enrolled in an introductory psychology class at the Ohio State University. All subjects reported they were in good health.

\section{Apparatus}

The subjects were tested individually in a sound-attenuated and electrically shielded chamber. The stimuli were projected on a screen in the chamber by a Kodak Carousel slide projector programmed for automatic advance and stimulus presentation with BRS Foringer digibit logic units. An intercom system connected the experimental chamber and the control chamber so that the experimenter could continuously hear the subject and could communicate with him or her when necessary. Physiological recording was done with a Grass Model 7B polygraph equipped with appropriate bridges, preamplifiers, and driver amplifiers. The voltage or resistance measures were written out on Grass ink-writing oscillographs providing graphic representation of the physiological activity.

Heart rate, endosomatic skin potential (GSP), and respiration were monitored for all 20 subjects. The respiratory results were not analyzed because of large between-subject variability in respiratory rate and depth. In addition, galvanic skin response (GSR) and cephalic pulse amplitude (CPA) were monitored for three subjects in each of the four conditioning groups.

\section{Electrode Placement}

Heart rate. Grass E5S cup electrodes filled with EKG Sol were placed on the lower left rib cage and the right collar bone. The signal was amplified by a Grass wide-band ac preamplifier and averaged by a Grass cardiotachometer which provided a beat-bybeat record of the heart rate.

Endosomatic GSP. Lowstanding potential (less than $50 \mu \mathrm{V}$ ), low dc drift, $\mathrm{Ag}-\mathrm{AgCl}$ Beckman electrodes were attached to the volar surface of the second phalange of the second finger of the nonpreferred hand (active electrode) and the inner surface of the nonpreferred forearm (reference electrode). The electrode sites were swabbed with acetone to remove oils and activity at the reference site was abolished by brisk rubbing with a gauze pad. The electrode medium was $.05 \mathrm{M} \mathrm{NaCl}$ in an inert plastic medium. The difference in the standing potentials at the two sites was amplified by a one megohm input impedence Grass Model 7P1 dc preamplifier and written on the Grass oscillograph. A bucking voltage of opposite polarity supplied by the preamplifier balanced the dc potential and kept the oscillograph on channel.

Exosomatic GSR. A curved $\mathrm{Ag}-\mathrm{AgCl}$ electrode of $3 \mathrm{~cm}^{2}$ placed on the volar surface of the second phalange of the preferred hand served as the active electrode and a curved armband of $58 \mathrm{~cm}^{2}$ placed on the upper arm served as the inactive electrode of a monopolar placement. A constant current of $20 \mu \mathrm{A}$ supplied by a Con-Sol GSR preamplifier was impressed at the active site making that portion of the subject's epidermis lying between the active and inactive electrodes an arm of a resistance bridge. A bandage with a $2-\mathrm{cm}^{2}$ hole in it restricted the current density to $10 \mu \mathrm{A} / \mathrm{cm}^{2}$ at the active electrode, whereas the current density at the reference electrode was $.314 \mu \mathrm{A} / \mathrm{cm}^{2}$. A $.05 \mathrm{M} \mathrm{NaCl}$ solution suspended in an inert plastic medium served as electrode paste. Surface body oils were removed by swabbing the electrode sites with acetone.

Cephalic pulse amplitude. A "cool" reflective photoelectric plethysmograph, comprising three GaAsP light-emitting diodes (LEDs) rediating $100 \mu \mathrm{W}$ of light output at $660 \mathrm{~nm}$ was employed. ${ }^{1}$ The LEDs were spaced at $120^{\circ}$ on a radius of $1 / 4 \mathrm{in}$. around a CdSe high-speed photoconductor. The transducer was placed over the supraorbital notch, providing a relative measure of blood volume in the supraorbital artery, a branch of the opthalmic artery (Wallace \& Wallace, 1968). Assessment of CPA was accomplished by ac coupling the response at $.1 \mathrm{sec}$.

\section{Procedure}

The subject was greeted by the experimenter and was given the APQ. The subject was then taken to the experimental cubicle, placed in a reclining chair, and asked to relax. Electrodes for monitoring the physiological indices were attached. A 10-min rest period then followed, allowing the subject to adapt to the laboratory. During the rest period, the subject's overall APQ score was determined and the subject was assigned to the high or low APQ group.

At the end of the 10-min rest period, the experimenter reentered the experimental cubicle and read the instructions aloud. All subjects were told that the purpose of the experiment was to determine to what degree individuals could learn to control certain aspects of their physiological responding. The subjects were informed that a series of red and green slides would be projected on a viewing screen in the experimental chamber and that they were to try to produce "correct" physiological responses when these slides were presented. Half of the subjects in both the high and low APQ groups were told that two different physiological responses were to be produced during stimulus presentation, but were not told that the correct responses were heart-rate acceleration and deceleration (uninformed group). The other half of the subjects were told that they would have to produce heart-rate accelerations to one stimulus and heart-rate decelerations to the other stimulus (informed group). Subjects in the informed group were further instructed to try to produce only heart-rate changes and not to change other physiological responses.

All subjects were given feedback contingent on their heart rates by means of a small, real-time clock. The clock ran continuously as along as the subjects produced responses of criterion magnitude during conditioning trials. The subjects were told that the person who accumulated the most time on the clock would be contacted when the experiment was over and paid \$20. All subjects were instructed to remain as still as possible and not to change their respiratory patterns. The subjects were told that if the experimenter 
noted radical changes in respiration he would ask them to try to breathe normally.

\section{Conditioning Procedure}

All conditioning trials were $\mathbf{3 0}$ heart interbeat intervals (IBIs) in length and were separated from each other by a variable intertrial interval of 10 to $30 \mathrm{sec}$. The order of stimulus presentation was randomized, with the restriction that the same color stimulus could not occur on more than three successive trials. Following the instructions was a brief rest period during which a basal heart rate was determined by counting the number of heart beats in a 1-min sample. This sample constituted the basis for the initial acceleration and deceleration criteria.

The heart-rate signal from the cardiotachometer was fed to two electronic circuits (BRS Foringer level detectors, Model 210) which detected changes in the voltage level of the cardiotachometer. These circuits automatically controlled the delivery of the reinforcement (turned the clock on and off). One circuit was used during acceleration trials and the other during deceleration trials.

An operant shaping procedure was employed during the conditioning trials. Prior to the first conditioning trial, the voltage detectors were set at 2 beats per minute above and below the subject's basal heart rate. For the first acceleration trial, the subject had to produce a heart rate 2 or more beats per minute (bpm) above his base rate to obtain reinforcement, while during the first deceleration trial the reinforcement criterion was 2 or more beats per minute below his base rate.

On subsequent conditioning trials, the reinforcement criteria depended on how well or how poorly the subject had done on the preceding trial. If a subject produced 22 or more IBIs of criterion magnitude during one trial (i.e., obtained reinforcement during more than $70 \%$ of the IBIs), the criterion for the next trial with the same stimulus was made more difficult by raising or lowering the heart-rate level $2 \mathrm{bpm}$. Conversely, if a subject produced eight or fewer "correct" IBIs during a trial (i.e., obtained reinforcement during fewer than $30 \%$ of the IBIs), the criterion for the next trial with the same stimulus was made $2 \mathrm{bpm}$ easier. All subjects were given $\mathbf{4 0}$ conditioning trials. Half of these trials were acceleration trials and half were deceleration trials.

\section{Data Reduction}

Heart rate. The length of each trial was measured to the nearest millimeter on the oscillograph record. Since each trial was 30 IBIs long, the length of the trial was determined by the subject's average heart rate during the trial. Since the paper speed for all subjects was $15 \mathrm{~mm} / \mathrm{sec}$, the average heart rate in each trial was defined by the formula $\mathrm{HR}=30 \times[(15 \mathrm{~mm} / \mathrm{sec} \times$ $60 \mathrm{sec}) / Y \mathrm{~mm}$ ], where $Y$ is the length of the trial in millimeters. This formula was used to determine the subject's average heart rate for each trial.

A similar procedure was employed to determine the subject's average heart rate during the $10 \mathrm{IBI}$ immediately preceding each trial. For the pretrial, the formula defining the average heart rate was $\mathrm{HR}=10 \times[(15 \mathrm{~mm} / \mathrm{sec} \times 60 \mathrm{sec}) / \mathrm{Y} \mathrm{mm}]$.

Endosomatic skin potential. Two types of GSP responses were scored during each trial and pretrial period:

(1) The number of negative pen deflections in which the angle of incline was greater than the angle of decline was tabulated. This type of response has been termed a "tonic" response by Kaiser and Sandman (1975) and Miller (1967).

(2) A "phasic" response was described as a negative pen deflection in which the angle of inflection was equal to the angle of deflection, or in which the negative pen deflection was followed by a positive deflection, or a positive pen deflection (Kaiser \& Sandman, 1975; Miller, 1967). The number of phasics was tabulated.

Exosomatic GSR. The number of GSR responses greater than 100 ohms was tabulated during each trial and pretrial and converted into responses per second by dividing by the amount of time during the trial or pretrial.
Cephalic pulse amplitude. The heights (in millimeters) of the largest cephalic pulse wave and the smallest pulse wave were measured and averaged for each pretrial period. For each trial, the heights (in millimeters) of the two largest pulse waves and the two smallest waves was measured and averaged.

\section{RESULTS}

\section{Heart Rate}

A 2 by 2 by 2 by 2 by 20 analysis of variance with repeated measures over the last three factors was computed using the average heart rate during each trial and pretrial period. For the purposes of this analysis, subjects were classified as having high or low APQ scores based on their total APQ score. This analysis yielded a significant Trials effect $(\mathrm{F}=16.47 ; \mathrm{df}=19,304 ; \mathrm{p}<.001)$, a significant Training by Response Sample interaction $(F=10.73$; $\mathrm{df}=1,16 ; \mathrm{p}<.01$ ), and a significant Training by Instructions by Response Sample interaction $(\mathrm{F}=$ 11.94; $\mathrm{df}=1,16 ; \mathrm{p}<.01)$. The highly significant Trials effects is illustrated in Table 1 . As Table 1 indicates, informed and uninformed subjects exhibited decreases in heart rate over training trials during both acceleration and deceleration conditioning. This generalized decrease in heart rate was probably attributable to adaptation to the laboratory setting and procedures. No significant interactions were noted between the trials factor and the other independent variables, which indicated that the adaptation of the heart-rate response was not affected by the experimental manipulations.

The significant three-way interaction detected by the analysis of variance is illustrated in Figure 1. Separate analyses of variance for uninformed and informed subjects indicated a significant Training by Response Sample interaction for informed subjects $(F=14.38 ; \mathrm{df}=1.8 ; \mathrm{p}<.01)$ but not for uninformed subjects $(\mathrm{F}<1 ; \mathrm{df}=1,8)$. For uninformed subjects, however, a significant Response Sample main effect was detected $(F=5.96 ; \mathrm{df}=1,8 ; \mathrm{p}<.05)$. Inspection of Figure 1 indicated that info - subjects produced pretrial-to-trial heart-raiv acceleration during acceleration training and pretrial-to-trial heart-rate deceleration during deceleration training. However, uninformed subjects produced pretrial-totrial deceleration during both acceleration and

Table 1

Average Heart Rate During Acceleration and Deceleration Training for Informed and Uninformed Subjects

\begin{tabular}{lcccc}
\hline & \multicolumn{2}{c}{ Trials $1-10$} & \multicolumn{2}{c}{ Trials $11-20$} \\
& Pretrial & Trial & Pretrial & Trial \\
\hline & \multicolumn{4}{c}{ Informed Subjects } \\
Acceleration Training & 79.7 & 82.4 & 75.3 & 77.3 \\
Deceleration Training & 80.2 & 78.6 & 75.4 & 74.9 \\
& \multicolumn{4}{c}{ Uninformed Subjects } \\
Acceleration Training & 82.5 & 80.2 & 77.3 & 76.1 \\
Deceleration Training & 81.6 & 79.4 & 77.0 & 75.9 \\
\hline
\end{tabular}




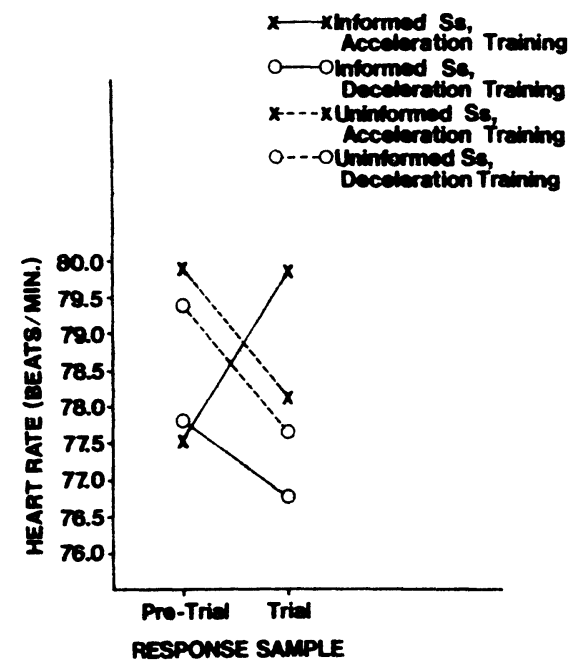

Figure 1. Pretrial-to-trial changes in average heart rate.

deceleration training. As Figure 1 indicates, uninformed subjects exhibited higher resting heart rates than informed subjects during the pretrial periods preceding both acceleration and deceleration conditioning trials. This result suggested that uninformed subjects may have produced the decelerative changes in heart rate illustrated in Figure 1 simply because they had reached a ceiling in heart rate during pretrial periods. That is, uninformed subjects may not have been able to produce pretrial-to-trial increases in heart rate because their heart rates were relatively high to begin with. This explanation suggested that the lack of information about response-reinforcement contingencies may have produced more "arousal" in the heart-rate modality than relatively detailed information about contingencies of reinforcement.

However, a separate analysis of variance of pretrial heart-rate values failed to indicate a significant difference between informed and uninformed subjects $(F=0.22, \mathrm{df}=1,16)$. Thus, although the explanation outlined above seemed intuitively obvious, it was not supported by a post hoc analysis of pretrial heart-rate levels.

The pretrial-to-trial deceleration in heart rate noted for uninformed subjects during acceleration and deceleration conditioning suggested that increases in heart rate were more difficult for these subjects to produce than decreases in heart rate. It seemed conceivable that uninformed subjects may have needed more than 20 conditioning trials in order to learn to produce increases in operant heart rate. If increases in operant heart rate were more difficult for subjects to learn, then informed subjects should have exhibited larger increases during later. training trials than during earlier trials. However, the data in Table 1 indicated that informed subjects were able to produce operant heart-rate increases throughout conditioning, thus demonstrating that operant heart-rate acceleration was relatively difficult for uninformed subjects but not for informed subjects.

Because the initial analysis of variance indicated no main effects or interactions attributable to overall APQ score, two additional analyses of variance were computed using different methods of scoring the APQ and classifying the subjects into two groups. ${ }^{2}$ For the first of these analyses, the five items on the APQ that pertain to heart-rate functioning were scored and informed and uninformed subjects were split into high and low APQ groups separately based on this score. (This method of scoring is identical to that reported by Blanchard et al., 1972, and McFarland, 1975). For the second of these analyses, the overall APQ score was utilized, but subjects were split into two groups based on the extremity of their APQ score. For uninformed subjects, the mean overall APQ score was computed. The differences between each subject's APQ score and the mean APQ score was then computed. The two subjects with the largest positive difference scores and the two subjects with the largest negative difference scores were classified as having extreme APQ scores. From the other six subjects, the subject with the largest difference score, regardless of sign, was also classified as having an extreme APQ score. The remaining five subjects were classified as having middle APQ scores. The same procedure was repeated for informed subjects. (This method of classifying subjects into groups is similar to that used by Bergman \& Johnson, 1971). Separate 2 by 2 by 2 by 2 by 20 repeated-measures analyses of variance were computed for the heart-rate data using each of the techniques for scoring APQ.

Both of these analyses of variance yielded results similar to those noted above. In both analyses, a significant Trials main effect $(p<.001$ for each analysis), a significant Training by Response Sample interaction ( $\mathrm{p}<.01$ for each analysis), and a significant Instructions by Training by Response Sample interaction ( $p<.05$ for each analysis) were detected. Neither of the analyses indicated a significant main effect or interaction due to APQ score. These results indicated that a subject's score on the APQ was not related to control over operant heart-rate, although the information subjects received about the contingencies of reinforcement did have significant effects on operant heart-rate control.

\section{GSP Responses}

Separate repeated-measures analyses of variance were also computed for the number of GSP tonic responses and the number of GSP phasics. Only the results using overall APQ scores divided into high and low groups will be reported, since the analyses using other APQ scoring methods were quite similar to the overall results. The analysis of the 


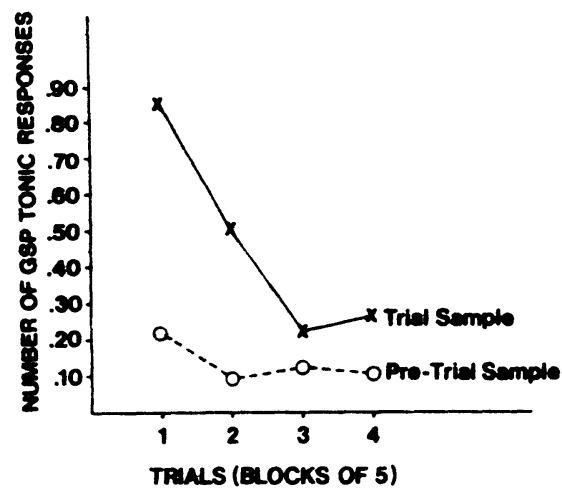

Figure 2. Pretrial-to-trial changes in GSP tonic responding.

number of GSP tonic responses yielded a significant Trials effect $(F=5.53 ; \mathrm{df}=19,304 ; p<.001)$, a significant Response Sample effect $(F=18.54$; $\mathrm{df}=1,16 ; \mathrm{p}<.001$ ), and a significant Trials by Response Sample interaction $(F=3.27$; $d f=19,304$; $\mathrm{p}<.01)$. The significant interaction is illustrated in Figure 2.

As Figure 2 indicates, there were substantial pretrial-to-trial increases in tonic responding during the early stages of training, but the magnitude of the increases declined during the latter stages of training. It seemed likely that the initial increases in tonic responding may have been part of a larger orienting response which habituated over trials.

The analysis of variance of the number of GSP phasics indicated a significant main effect for Training $(F=7.22 ; \mathrm{df}=1,16 ; p<.05)$. No other significant main effects or interactions were detected. The main effect indicated that more phasic responding occurred during acceleration training $(M=.063)$ than during deceleration training $(\mathbf{M}=.029)$.

\section{GSR Responses}

A five-factor analysis of variance with repeated measures over the last three factors was computed for those subjects from whom GSR data were available. This analysis yielded a significant Instructional Set main effect $(F=7.09 ; \mathrm{df}=1,8 ; \mathrm{p}<.05)$. No other significant main effects or interactions were detected. The main effect indicated that informed subjects produced significantly more GSR responses $(\mathrm{M}=.139 \mathrm{responses} / \mathrm{sec})$ than uninformed subjects $(\mathbf{M}=.038 \mathrm{responses} / \mathrm{sec})$ during the experiment.

\section{Cephalic Pulse-Amplitude}

Because the cephalic plethysmograph yielded a relative rather than absolute measure of blood flow, pretrial-to-trial changes in CPA were computed for each subject for each trial. The change for each trial was then divided by the pretrial value for each subject, yielding a measure of percentage change for each trial. The percentage change figures were then subjected to a 2 by 2 by 2 by 20 analysis of variance with repeated measures over the last two factors. Thus, for this analysis, the Response Sample factor was omitted.

The repeated-measures analysis of variance for CPA yielded no significant main effects or interactions.

\section{DISCUSSION}

The heart-rate results of the present study indicated that an individual's awareness of the responsereinforcement contingencies (as manipulated by instructional set) was a stronger determinant of operant heart-rate control than proprioceptive awareness (as measured by the APQ). None of the techniques for scoring the APQ resulted in significant changes in heart rate which were due to proprioceptive awareness. The equivocal $A P Q$ results reported in previous studies (Bergman \& Johnson, 1971; Blanchard et al., 1972; McFarland, 1975; Sirota et al., 1974) and the lack of significant differences in operant heart-rate control due to APQ scores reported in the present study raise serious questions about the relationship between questionnaire measures of proprioceptive awareness and control over autonomic responses. It seems likely that a behaviorally derived measure of proprioceptive awareness may be more directly related to autonomic control than a questionnaire measure such as the APQ.

The instructional set of subjects had not been varied in previous experiments involving the APQ. However, contrary to expectations, the results did not indicate that APQ score was important in determining operant heart-rate control when subjects were not informed of the specific response-reinforcement contingencies.

It should be noted that the present study utilized procedures quite different from those employed by the experimenters who have noted differences in heart-rate control due to APQ score (Bergman \& Johnson, 1971; Blanchard et al., 1972). The most notable difference between this study and the report of Bergman and Johnson (1971) was the addition of operant reinforcement in the present experiment. Bergman and Johnson's (1971) results indicated that, in the absence of external feedback, subjects with middle APQ scores obtained better control over heart rate than subjects with extreme scores. However, when external feedback was available to subjects (as in the present study), there appeared to be no differences in heart-rate control between APQ groups.

There were also several procedural differences between the present study and the report by Blanchard et al. (1972). While visual feedback was utilized in both experiments, the feedback in the present experiment had monetary consequences associated with it, while no money was associated 
with the feedback in the Blanchard et al. (1972) study. When a relatively weak reinforcer (such as visual feedback) is utilized to produce changes in operant heart rate, proprioceptive awareness may play an important role in determining the amount of heart-rate control a subject achieves. However, when a relatively powerful reinforcer (such as visual feedback with monetary consequences) is used in conditioning changes in operant heart rate, the proprioceptive awareness of the subjects appears unrelated to heart-rate control.

This interpretation is consistent with the results reported by Sirota et al. (1974), which indicated no effect of APQ score on operant heart-rate control using monetary reinforcement. However, the interpretation is contradicted somewhat by McFarland's (1975) study, which suggested that there were no significant relationships between APQ score and heart-rate control when simple visual feedback was employed. McFarland's (1975) suggestions were based on correlational analyses, however, rather than on the experimental manipulations 'of APQ scores utilized in the other studies.

The decrease in operant heart rate noted for uninformed subjects during both acceleration and deceleration training may have resulted because these subjects exhibited higher resting heart rates than did informed subjects between trials. Thus, the uninformed subjects may have been more "aroused" than informed subjects by the instructions they were given. However, a post hoc analysis indicated that the pretrial heart rates of uninformed subjects did not differ significantly from those of informed subjects. Moreover, none of the other physiological variables reflected greater "arousal" for uninformed subjects than for informed subjects. In fact, the GSR results indicated that informed subjects were more "aroused" than uninformed subjects.

Operant heart-rate acceleration appeared to be relatively difficult for uninformed subjects to produce, but informed subjects were able to produce increases in operant heart rate even during early training trials. However, it should be noted that uninformed subjects can produce significant increases in operant heart rate when they are given extended training over several sessions (McCanne $\&$ Sandman, 1975b). Thus, the 20 acceleration training trials of the present experiment may not have been enough for uninformed subjects to learn to produce increases in operant heart rate.

The Laceys and their colleagues (Lacey, 1967; Lacey \& Lacey, 1970, 1974; Lacey, Kagan, Lacey, $\&$ Moss, 1963) have noted decreases in heart rate during tasks that demand attention to environmental stimuli. It seems likely that the pretrial-to-trial decreases in heart rate noted for uninformed subjects may have resulted simply because they were attending to the discriminative stimulus and the reinforcing stimulus. Thus, when subjects are given relatively undetailed instructions about the contingencies of reinforcement, the changes in heart rate that occur for those subjects may be a result of the attentional demands of the conditioning procedure.

The skin potential results suggested that heart-rate acceleration training was accompanied by more sympathetic-like skin responses (as indicated by GSP phasic responding) than deceleration training. These results appear to be somewhat contradictory to those reported in an earlier study (McCanne \& Sandman, 1976b), which indicated that changes in operant heart rate were relatively independent of changes in skin potential. However, our earlier study examined physiological responses over several conditioning sessions. In addition, the analyses of GSR responding and cephalic pulse amplitude failed to indicate any significant differences in responding between acceleration and deceleration training. Moreover, none of the analyses of GSP responding, GSR responding, or cephalic pulse amplitude indicated any type of complex interaction paralleling the complex interactions noted for operant heart rate. The results of these analyses indicate that the changes in operant heart rate were relatively independent of changes in other autonomic variables even within a single conditioning session.

\section{REFERENCES}

Bergman, J. S., \& Johnson, H. J. The effects of instructional set and autonomic perception on cardiac control. Psychophysiology, $1971,8,180-190$.

Bergman, J. S., \& Johnson, H. J. Sources of information which affect training and raising of heart rate. Psychophysiology, 1972, 9, 30-39.

Blanchard, E. B., Young, L. D., \& McLeod, P. Awareness of heart activity and self-control of heart rate. Psychophysiology, 1972, 9, 63-68.

Kaiser, D. N., \& Sandman, C. A. Physiological patterns accompanying complex problem solving during warning and nonwarning conditions. Journal of Comparative and Physiological Psychology, 1975, 89, 357-363.

LACEY, J. I. Somatic response patterning and stress. In M. H. Appley \& R. Trumball (Eds.), Psychological stress: Issues in research. New York: Appleton-Century-Crofts, 1967.

Lacey, J. I., Kagan. J., LACEY, B. C., \& Moss, H. A. The visceral level: Situational determinants and behavioral correlates of autonomic response patterns. In P. Knapp (Ed), Expressions of the emotions in man. New York: International Universities Press, 1963.

LACEY, J. I., \& LACEY, B. C. Some autonomic-central nervous system inter-relationships. In P. Black (Ed.), Physiological correlates of emotion. New York: Academic Press, 1970.

LACEY, J. I., \& LACEY, B. C. On heart rate responses and behavior: A reply to Elliott. Journal of Personality and Social Psychology, 1974, 30, 1-18.

Mandler, G., Mandler, J. M., \& Uviller, E. T. Autonomic feedback: The perception of autonomic activity. Journal of Abnormal and Social Psychology, 1958, 58, 367-373. 
McCanne, T. R., \& Sandman, C. A. Determinants of human operant heart-rate conditioning: A systematic examination of several methodological issues. Journal of Comparative and Physiological Psychology, 1975, 88, 609-618. (a)

McCanne, T. R., \& Sandman, C. A. The impact of two different reinforcers on conditioned operant heart-rate acceleration and deceleration. Biological Psychology, 1975, 3, 131-142. (b)

MCFARLAND, R. A. Heart rate perception and heart rate control. Psychophysiology, 1975, 12, 402-405.

MILLER, L. H. The bidimensional nature of the galvanic skin response. Unpublished doctoral dissertation, Duke University, 1967.

Sirota, A. D., Schwartz, G. E., \& Shapiro, D. Voluntary control of human heart rate: Effect on reaction to aversive stimulation. Journal of Abnormal Psychology, 1974, 83, 261-267.

Wallace, S., \& Wallace. J. D. Hemodensitometry in the evaluation of cerebral circulation. Radiology, 1968, 90, 454-465.

\section{NOTES}

1. The cephalic plethysmograph was developed by Robert Isenhart.

2. It should be noted that these analyses of variance were conducted post hoc. Neither the score of the five APQ heartrate items nor the extremity of the subject's overall APQ score was manipulated experimentally in the original design of the experiment. However, the post hoc analyses were conducted to determine whether other methods of scoring the APQ may have resulted in significant main effects or interactions due to autonomic awareness. Because the analyses were conducted post hoc, the results must be interpreted with caution.

(Received for publication December 12, 1975; revision accepted April 12, 1976.) 